\title{
Advances in abdominal access for laparoscopic surgery: a review
}

This article was published in the following Dove Press journal:

Open Access Surgery

19 November 2014

Number of times this article has been viewed

\author{
Maurice Ogaick' \\ Guillaume Martel ${ }^{1,2}$ \\ 'Department of Surgery, ${ }^{2} O$ ttawa \\ Hospital Research Institute, University \\ of Ottawa, Ottawa, ON, Canada
}

Correspondence: Guillaume Martel Liver and Pancreas Unit, The Ottawa Hospital, 50I Smyth Rd, Ottawa,

ON, Canada

$\mathrm{Tel}+$ I 6137378899

Fax + I6I37396854

Email gumartel@ottawahospital.on.ca
Abstract: There are several laparoscopic surgery abdominal wall access techniques. The most useful and well-established is the open Hasson technique. The Veress needle closed technique is another alternative, but its use is controversial owing to possibly higher rates of rare insertion complications. Optical trocars have been developed in an attempt to decrease complication rates even further, although the evidence base supporting their use is limited. Single-incision laparoscopic surgery (SILS) is used in certain centers to carry out various abdominal surgeries, but most commonly appendectomy and cholecystectomy. To date, SILS appears safe and feasible, with possibly greater costs and operative time. Natural orifice translumenal endoscopic surgery (NOTES) is an evolving area of incisionless surgery. While some progress has been made in achieving transgastric or transvaginal peritoneal endoscopic access, this technique remains largely unproven and best-suited for experimental or clinical trial protocols.

Keywords: laparoscopy, Hasson, Veress, optical trocar, SILS, NOTES

\section{Introduction}

Over the last 20 years, laparoscopy has emerged as one of the primary operative techniques within the sphere of abdominal surgery. During this time, it has undergone significant changes as new technologies and operative approaches have become available. The application of these has allowed laparoscopic surgery to be applied to ever more complex operations, such as liver resections and pancreaticoduodenectomies. Besides offering smaller incisions, and thus often a better cosmetic result, laparoscopic surgery has also been shown to decrease postoperative pain, reduce the time required to return to work, and reduce the number of wound infections and hernias compared to the more traditional open technique. ${ }^{1}$

Despite these encouraging results, complications do occur during laparoscopic surgery, many of which take place during initial entry into the peritoneal cavity. This has led to the development of several techniques for entry, including the open Hasson method, the Veress needle method, and the use of specialized instruments such as optical trocars. In addition, the evolution of laparoscopy has led to the development of novel, progressively less invasive approaches, including single-incision laparoscopic surgery (SILS) and natural orifice translumenal endoscopic surgery (NOTES) in an effort to minimize or eliminate abdominal incisions and their related complications.

This paper will discuss both the currently available laparoscopic access techniques, as well as the newer SILS and NOTES techniques, including their application, efficacy, and complications. 


\section{Hasson technique}

The Hasson technique refers to one method of accessing the abdomen for laparoscopic surgery via an open approach. In this method, a small, $1.5-2 \mathrm{~cm}$, incision is made either inferior or superior to the umbilicus when the patient is supine. The incision itself may be in a vertical or horizontal direction depending on the surgeon's preference. The incision is then extended down through the subcutaneous tissues until the abdominal fascia can be grasped and elevated with an appropriate instrument, such as a Kocher forceps. Visualization of the fascia can be facilitated with the use of retractors, such as an S-retractor or Langenbeck. In patients with a greater amount of subcutaneous tissue, it may also be necessary to grasp the umbilical stalk and tent it superiorly in order to bring the fascia into view. ${ }^{2}$

Once the fascia has been grasped on either side of the midline, it is divided sharply for $1-2 \mathrm{~cm}$ with a scalpel. This should allow for visualization and grasping of the peritoneum with a smaller instrument, such as snap or Kelly. The peritoneum is then incised under direct visualization. With the peritoneum open, the surgeon inserts a finger or blunt instrument, such as a Kelly clamp, to ascertain whether or not access has truly been achieved. If no adhesions or resistance are felt, an anchoring stitch can be placed on either side of the incision to aid both in securing the access trocar and closing the incision at the end of the case. Alternatively, stitches may be placed in the fascia prior to the incision.

Provided there is no evidence of adhesions or injury to underlying organs, an appropriate laparoscopic trocar is introduced into the abdomen. The traditional Hasson trocar, or cannula, consists of three separate pieces: an outer sheath, a cone-shaped attachment, and blunt introducer (obturator). ${ }^{3}$ The blunt introducer allows for safe advancement of the cannula into the abdominal cavity, and the cone-shaped attachment can be anchored to the skin incision to prevent leakage of carbon dioxide and subsequent loss of pneumoperitoneum. In addition, the cone-shaped attachment can be adjusted along the sheath to accommodate for the length of cannula required to access the abdomen. The cannula may also be further secured by wrapping the anchoring stitches around specialized struts on either side of the sheath.

To introduce the Hasson cannula, the fully assembled device should be advanced into the peritoneal cavity with the blunt introducer in place. Once an adequate length of cannula is inside the abdomen, the cone-shaped attachment is moved down to the skin incision and secured to the sheath. The anchoring stitches are then tightened onto the struts to further secure the trocar.
If a true Hasson trocar is not available, the same entry technique may be used with a variety of trocars, including disposable and reusable alternatives. These may not have the additional cone-shaped attachments and struts, but the technique of open access into the abdominal cavity remains the same.

Once the access trocar is secure, the abdomen is insufflated to a pressure of $15 \mathrm{mmHg}$. It is important to carefully watch the gas insufflator as pneumoperitoneum is achieved, as it may provide valuable information regarding the trocar's placement. Ideally, gas should flow through a $12 \mathrm{~mm}$ port at approximately $10 \mathrm{~L} /$ minute, and the pressure reading should slowly increase until the desired settings are reached. Approximately 3-6 L of gas are required to insufflate the standard abdomen. Initially, the measured pressure in the abdomen should be low (3-4 mmHg), though this may be closer to $8 \mathrm{mmHg}$ in obese patients.

After the desired pneumoperitoneum is achieved, the surgeon introduces a laparoscope and performs a laparoscopy. The laparoscope is initially introduced vertically so that any underlying structures, such as bowel or omentum, can be examined for injuries sustained during initial access. Provided no injury has occurred, it is safe to proceed with the proposed laparoscopic procedure.

Although the open technique has been described here for supra- and infra-umbilical access, it may be used for essentially all port placement locations. As it relies on direct visualization of the fascia and peritoneum during entry, the Hasson technique is often cited as being the safest of entry techniques. In particular, it is well-suited for use in patients with a history of previous abdominal surgery or other conditions that may result in intra-abdominal adhesions. Once pneumoperitoneum has been safely achieved, nearly all procedures can be completed laparoscopically.

Unfortunately, laparoscopic surgery is not without complications, and the majority of these complications occur during initial entry into the abdomen. Complications may arise from the fascial incision, peritoneal division, or the introduction of the obturator and laparoscope. Regardless of the mechanism of injury, complications can be categorized as either minor or life-threatening. Minor complications include superficial wound infections, subcutaneous emphysema, and abdominal wall hematomas. Major, life-threatening complications include injuries to the bowel, bladder, and major vasculature of the abdominal cavity, abdominal wall, or retroperitoneum. Such complications may result in conversion of a laparoscopic procedure to open, reoperation, and significant morbidity and mortality. ${ }^{2}$ 
Despite the potential for serious harm, complications relating to laparoscopic access are relatively rare (Table 1). In one review of the literature, bowel perforation was reported in 1.8 per 1,000 cases $(0.18 \%)$ and major vessel injury at 0.9 per $1,000(0.09 \%)$ cases. $^{2}$ In Hasson's initial assessment, the rate of wound infection was also low at $0.4 \% .{ }^{4}$ Mortality is also extremely low with several studies suggesting a rate approaching $0 \%{ }^{4}$ In his own retrospective series, Hasson noted complications related to open access in $0.5 \%$ of 5,284 patients..$^{5}$ Of these complications, the majority were wound infections or minor hematomas. ${ }^{5}$ Only one patient developed an umbilical hernia that required reoperation, and only one patient had an inadvertent small bowel injury that was repaired at the time of the operation. ${ }^{5}$ There were no life-threatening complications. Multiple investigators from both General Surgery and Gynecology have generated similar results, suggesting the open technique of laparoscopic access to be extremely safe and the access technique of choice.

\section{Veress needle/closed technique}

Alternatively, some surgeons opt to perform a "closed" form of laparoscopic access using a specialized needle to puncture the abdomen. This needle, known as a Veress needle, is an approximately 14-gauge needle that is composed of a sheath and a retractable blunt tip. Disposable versions of the needle also exist. ${ }^{3}$

The reusable Veress needle should be checked prior to usage by retracting the sheath and ensuring that it springs back into place when released. This ensures that the blunt tip will retract appropriately when it penetrates the various layers of the abdominal wall.

In order to use the closed technique, the patient is placed in the supine position and a small incision made either superior or inferior to the umbilicus. The subcutaneous tissues are bluntly pushed aside using gauze, and the umbilicus is then grasped with a retractor and elevated. Alternatively, two stay stitches can be placed into the fascia directly. This provides some counterattraction so that the fascia is taut when the Veress needle is inserted. The needle is classically inserted at either

Table I Comparison of complications with traditional access techniques

\begin{tabular}{llll}
\hline & $\begin{array}{l}\text { Overall } \\
\text { complications }\end{array}$ & $\begin{array}{l}\text { Bowel } \\
\text { injury }\end{array}$ & $\begin{array}{l}\text { Vascular } \\
\text { injury }\end{array}$ \\
\hline $\begin{array}{l}\text { Open Hasson } \\
\text { technique }\end{array}$ & $0.5 \%$ & $0.048 \%-0.1 \%$ & $0 \%$ \\
$\begin{array}{l}\text { Closed Veress } \\
\text { technique }\end{array}$ & not available & $0.083 \%-0.2 \%$ & $0.044 \%-0.2 \%$ \\
\hline
\end{tabular}

a $45^{\circ}$ or $90^{\circ}$ angle. Ideally, there should be several "pops", or moments of resistance followed by give, as the needle passes through the fascia and then through the peritoneum. There is often a definitive "click" when the needle passes through the peritoneum and the blunt tip retracts. ${ }^{3}$

Several steps have been recommended in the literature in order to double check the correct placement of the Veress needle. To start, a $10 \mathrm{cc}$ syringe containing sterile saline is connected to the Luer lock of the needle. The needle is aspirated to inspect for blood, bowel content, or urine. Then, $5 \mathrm{cc}$ of sterile saline can be injected into the peritoneal cavity. This should flow without resistance, and ideally does not reaccumulate within the syringe if reaspiration is attempted. Next, the "drop test" is performed by detaching the syringe and instilling a drop of water at the end of the Luer lock. The drop of water should disappear rapidly if the needle is in the correct location. Finally, the needle should be freely mobile within the abdominal cavity, allowing for further advancement without appreciable resistance. ${ }^{3}$

In addition to the above maneuvers, perhaps the most useful test to ensure the Veress is in the correct location comes when it is hooked up to the $\mathrm{CO}_{2}$ insufflator. As the Veress needle has a relatively small diameter, flow of $\mathrm{CO}_{2}$ through the insufflator cannot exceed 3-4 mmHg. If the needle is in a good position, then flow should read approximately $3 \mathrm{mmHg}$ while the resistance remains low. If flow is minimal and resistance is high, then the needle is not within the peritoneal cavity. In obese patients, the initial resistance may read higher (7-8 mmHg), but flow should continue unimpeded.

Despite the fact that the Veress needle entry has a low complication rate, it is nevertheless considered more dangerous than the open technique. ${ }^{4}$ Similar to the open technique, major complications include bowel injury, major vascular injury, and wound infections (Table 1). In his initial report comparing the open to closed technique, Hasson determined that rate of the bowel and major vascular injury were $0.2 \%$ and $0.2 \%$, respectively. ${ }^{4}$ Although this is low, it is still higher than the $0.1 \%$ and $0 \%$ he reported for the open technique, respectively. ${ }^{4}$

This data has been reproduced and supported by several other studies. ${ }^{6-9}$ For example, in one large retrospective review from the Netherlands, 489,335 patients undergoing closed laparoscopy and 12,444 patients undergoing open laparoscopy were compared. ${ }^{6}$ The authors concluded that the open technique was safer and thus favored as there were no patient deaths or major vascular injuries reported from the open technique, compared to a $0.003 \%$ mortality and $0.075 \%$ major vascular injury rate in the closed technique. ${ }^{6}$ Rates of 
visceral injury were also low in both groups, but were still significantly less in the open technique group $(0.083 \%$ vs $0.048 \%) .{ }^{6}$ Larobina and Nottle showed similar results, with a vascular injury rate of $0.044 \%$ reported in the Veress needle group versus $0 \%$ in the open technique group. ${ }^{7}$

Interestingly, some authors have suggested that the Veress technique may result in decreased incisional hernia rates following laparoscopy. One retrospective review examined 747 patients undergoing laparoscopic Roux-en-Y gastric bypass and found a hernia rate of $1.20 \%$ in the Hasson port site (midline), whereas no hernias were detected in either $12 \mathrm{~mm}$ or $5 \mathrm{~mm}$ port sites placed via the Veress-based VersaStep trocar system (US Surgical, Norwalk, CT, USA). Despite the possibility of fewer incisional hernias, the Veress technique continues to have higher rates of visceral and vascular injury and should be considered inferior to the open technique.

\section{Optical trocars}

Although complication rates with both the open and Veress needle entry techniques are relatively low, they can nevertheless be devastating. Thus, efforts have been made to reduce them further. One such method involves placing the laparoscope directly within a sharp trocar during insertion, allowing the surgeon to directly visualize the various layers of the abdominal wall as the trocar is advanced. The specialized trocars created for this purpose are collectively known as optical trocars.

According to published reports, the various layers of the abdominal wall are easily identifiable as the optical trocar passes through them. The fat appears yellow or white, the muscle red, the fascia white, and the peritoneum relatively translucent. ${ }^{10}$ If there are no organs adherent to the peritoneum, a dark spot is seen beyond the tip of the trocar. ${ }^{10}$ Conversely, a whitish reflection is seen if adhesions are present. ${ }^{10}$ Unlike other sharp trocars, the optical trocar is designed to push away tissue in a radial direction rather than cut through it.

Various different optical trocars exist on the market, and the exact recommended technique for their insertion varies depending on the manufacturer. For example, the Endopath Xcel trocar (Ethicon Endo-Surgery, Somerville, NJ, USA) claims a bladeless optical tip and is positioned by slowly twisting the trocar into position. Similarly, the Versaport Bladeless Optical Trocar (Covidien, Mansfield, MA, USA) functions by dilating the tissue in a radial fashion under $0^{\circ}$ laparoscope visualization. In many instances, the manufacturer recommends using these trocars with a Veress needle insufflation technique.
Several investigators have studied the safety of optical trocars in practice. In their review of 650 patients undergoing a variety of general surgery procedures over a 4-year period, String et al demonstrated an extremely low complication rate. Only one injury to the bowel and one to the gallbladder were described, both of which were repaired immediately. Overall, the complication rate at entry was a remarkably low $0.3 \%{ }^{11}$ Both injuries occurred in patients with previous surgical scars.

These results were supported by a similar, smaller series by Hallfeldt et al. They analyzed 200 patients undergoing a variety of general surgery procedures, and aside from one wound infection, reported no complications from the use of optical entry trocars. Interestingly, adherent small bowel was visualized and avoided in four cases despite the selection of an entry site several centimeters from a previous incision site. $^{12}$

In addition to safety, both studies also analyzed the speed of entry into the abdomen and found them to be relatively rapid. In both series, the time to entry was rapid, with Hallfeldt et al reporting an average under 4 minutes and String et al between 1.5 and 2 minutes. ${ }^{11,12}$

\section{Single-port laparoscopy}

Single-port laparoscopy, or SILS, is an emerging form of minimally invasive surgery that relies on a single access point to perform a laparoscopic operation while minimizing scarring. It is still very much in its infancy and has not yet been adopted widely, but it has the potential to gain popularity as it uses modified laparoscopic instruments. This is in contrast with other novel techniques of minimally invasive surgery, such as NOTES, that require not only a steep learning curve, but also novel instruments and techniques. ${ }^{13}$

There are currently no agreed-upon standard techniques for the performance of SILS, but comprehensive review articles in the literature have summarized the various techniques available. Initial access to the abdominal cavity can be done in either the open or Veress method, as previously described. The location of the access incision varies, with some surgeons using umbilical access while others take an infraumbilical or periumbilical approach. Intra-abdominal pressure settings are also reported to be variable, ranging from $6 \mathrm{mmHg}$ to $11 \mathrm{mmHg} .{ }^{13}$ Once pneumoperitoneum has been achieved, the operation may be accomplished using a variety of surgical techniques and instruments. The number of incisions varies depending on the type of operation and the choice of intra-abdominal retraction device used. ${ }^{13}$ 
In the three-port technique, a single incision is made in the periumbilical skin and three separate ports are introduced via three separate fascial incisions. A combination of trocars has been reported, including the placement of one $5 \mathrm{~mm}$ and two $3 \mathrm{~mm}$ ports, or a larger $15 \mathrm{~mm}$ and two $3 \mathrm{~mm}$ ports. In theory, this technique should be the most similar to conventional laparoscopy, though the use of standard laparoscopic instruments does result in clustering of the handles. Some of these difficulties can be overcome by using more specialized equipment such as a deflecting laparoscope and articulating or angulated instruments. ${ }^{14,15}$

Similarly, a two-port technique has been successfully demonstrated in SILS cholecystectomy. In order to do this, a stay suture or similar retracting device is introduced through the skin and fascia in a different location. This device is then attached to the gallbladder, often at the fundus or Hartmann's Pouch, and gently pulled taut to serve as retractors. By doing so, one less port can be placed through the fascia at the periumbilical incision. ${ }^{13}$

A one-port access technique is also common and relies on a single large trocar that penetrates the fascia but has the ability to accommodate several additional channels. The size of the access port is variable. This technique is commonly used for SILS appendectomies, and a large number of different access devices have been created to accommodate them. These devices include the use of a standard surgical glove and a small Alexis (Applied Medical, Rancho Santa Margarita, CA, USA) wound retractor. In this case, the glove maintains pneumoperitoneum over the wound retractor, and the glove allows the introduction of a $5 \mathrm{~mm}$ laparoscope. Other instruments can then be inserted using other fingers ("slim pipes") to allow for dissection. ${ }^{16}$

Similarly, a GelPort (Applied Medical) hand-access device can be used as the principal access point, with room to introduce three separate trocars at varying distances from one another. ${ }^{17}$ The suppleness of the gel allows for flexibility and range of motion for the laparoscopic instruments, an advantage perceived by some compared to fixed systems with rigid parts. ${ }^{17}$ Other commercially available devices include the TriPort (Advanced Surgical Concepts, Bray, Ireland), the SILS Port (Covidien), and the SSL Access System (Ethicon EndoSurgery).${ }^{13}$ Although they are all slightly different, their general setup is the same. They all typically use a single incision with a wound retractor, which includes three integrated channels, or laparoscopic ports, one of which allows the introduction of a laparoscope, and two of which allow $5 \mathrm{~mm}$ instruments.

Numerous abdominal operations using SILS have been described. In their review of the literature, Froghi et al identified over 1,800 patients who underwent SILS. The vast majority of these patients underwent appendectomies, though cholecystectomies, sleeve gastrectomies, adjustable gastric banding, and inguinal hernia repairs have all been well-described. As the techniques have evolved, so too have the possible applications for SILS. Currently, nearly every surgery that can be accomplished laparoscopically can be attempted with SILS, including complex operations such as right hemicolectomies, proctocolectomies, and necrosectomies. ${ }^{13}$

Cholecystectomy is one of the more commonly performed operations using SILS, and requires several adjustments to accommodate for the decrease in triangulation through a single port. This can sometimes be accomplished with already existing laparoscopic equipment that reticulate, have right angles, or are bowed. ${ }^{18}$ Another useful technique is the insertion of a retraction device separately from the SILS port. Retraction devices can include "traction stitches" on the fundus and body of the gallbladder, a carefully placed endoloop on the fundus, or a hook-shaped Kirschner wire that can be bent into an appropriate shape. ${ }^{19-21}$

Appendectomies, too, can be accomplished through a variety of different techniques. In general, these can be divided into extra- or intracorporeal methods. In the extracorporeal technique, the appendix is visualized and grasped before being pulled into the SILS port. It can then be completely exteriorized as the abdomen is desufflated to allow for extracorporeal ligation. This is quite similar, in fact, to an open appendectomy. ${ }^{22}$ The appendix may also be ligated intracorporeally. This is technically more demanding, but can be accomplished with a "sling suture" which is passed from the abdominal wall through the mesoappendix to provide retraction. ${ }^{23}$ The appendix can then be dissected out and removed via the SILS port.

Despite the cosmetic advantages of using a single abdominal access point, SILS is still being evaluated in regards to its safety and efficacy compared to conventional laparoscopy. For cholecystectomy, the mean operative time appears comparable to conventional laparoscopy at 86.3 minutes (range 45-149 minutes). Complications were relatively rare, including a 5\% conversion to conventional laparoscopy rate (33/627 patients). Intraoperative complications such as gallbladder perforation and hemorrhage were rare. Postoperative complications were similarly scarce, and included urinary retention, wound infection, hematoma, and bile leaks. ${ }^{13}$ The conversion rate to conventional laparoscopy was somewhat higher in the group of patients undergoing appendectomy $(10 \%$ vs $5 \%)$. Despite this, postoperative complication rates 
were still small. Reported complications appear consistent with those of standard laparoscopy, including ileus, hemorrhage, abscess, and wound infections. ${ }^{13}$

Although some series report encouraging data, a recent randomized controlled trial (RCT) of 75 adult patients undergoing either SILS or conventional three-port laparoscopy raises questions about the overall utility of the novel technique. ${ }^{24}$ In their study, Carter et al demonstrated that SILS procedure took an average of $40 \%$ longer than conventional laparoscopy, while causing significantly more postoperative pain and opiate usage. ${ }^{24}$ Similar results regarding operative time were seen in another, smaller RCT in patients undergoing cholecystectomy. However, unlike the previous trial, there were no differences in postoperative pain or analgesic use. ${ }^{25}$

Overall, it seems that data regarding SILS are still somewhat mixed. Although the procedure is feasible and has a complication rate approaching that of standard laparoscopy, it is associated with longer operation times and increased cost, and therefore remains a largely experimental technique at the present time. ${ }^{26}$

\section{Natural orifice translumenal endoscopic surgery}

Although SILS has theoretically reduced the potential number of incisions for laparoscopic surgery to just one, the desire to completely eliminate surgical scars remains. As a result, some surgeons have looked for alternate sites to access the peritoneal cavity, including natural orifices such as the mouth, vagina, anus, and urethra. This idea originated as endoscopists observed that patients recovered fairly well after accidental puncture of visceral organs such as the stomach or colon during advanced endoscopic procedures. ${ }^{27-29}$

Aside from the cosmetic benefits of having no visible incision, there are several theoretical advantages that NOTES offers. For example, there may be decreased intra-abdominal adhesions, decreased hernia rates, decreased postoperative pain, shorter recovery time, and decreased surgical site infections. ${ }^{27-29}$ Unfortunately, many of these benefits remain unproven as few human studies exist in the literature to support them.

To date, a variety of different general surgical operations have been performed using NOTES. These include pancreatic necrosectomy, appendectomy, cholecystectomy, and peritonoscopy. ${ }^{30}$ In 2011, Clark et al described their experience with transgastric endoscopic pancreatic necrosectomy in 104 patients. They reported successful resolution in 95 of the patients, and eventually determined that transgastric debridement was both relatively safe and efficacious. ${ }^{30}$ Despite the success of this approach, their technique involved entering the closed space of the retroperitoneum rather than the abdominal cavity. The first appendectomy was reportedly done in India in 2006, but was not presented in the literature. ${ }^{30}$ In 2008, Hazey et al reported the first use of transgastric peritonoscopy. In their study, patients with pancreatic masses underwent both laparoscopy and NOTES peritonoscopy. Ultimately, they concluded that transgastric peritonoscopy was again both safe and efficacious. ${ }^{31}$

The techniques used to access the peritoneal cavity vary somewhat in the literature as they are still largely experimental. The most common access point is transvaginal, via the posterior fornix of the vagina, with approximately $79 \%$ of procedures being performed using this technique. ${ }^{30}$ This is most likely due to the fact that colpotomy and colpotomy closure are well-described procedures. In this technique, an endoscope is placed through the colpotomy and into the peritoneal cavity, which is insufflated to a desired pressure. Some investigators have used this combination with a transperitoneal incision in a hybrid laparoscopic/NOTES technique. ${ }^{32}$ Others have successfully placed a trocar and $5 \mathrm{~mm}$ retractors via separate colpotomies to achieve appropriate visualization of their target organs. ${ }^{33}$

One recent RCT by Bulian et al describes a similar transvaginal approach to cholecystectomy, but uses curved laparoscopic instruments instead of an endoscope for grasping and manipulating the gallbladder. ${ }^{34}$ In addition, a $5 \mathrm{~mm}$ trocar placed at the umbilicus is used for both clipping and dividing the cystic duct and artery. The gallbladder itself is then placed into a retrieval bag and delivered via one of the transvaginal ports. In their study, Bulian et al report significantly less postoperative pain using this technique compared to needlescopic cholecystectomy both at 2 and 10 days postoperatively using the Numeric Rating Scale (NRS)-11 ( 8 vs 14 on postoperative day 2, 22 vs 41 on postoperative day 10), as well as superior cosmetic results and quality of life scores. ${ }^{34}$ These benefits did not come at the expense of either operative time or, more importantly, patient safety. ${ }^{34}$

Although less commonly used, the majority of patients prefer a transgastric approach for NOTES. ${ }^{30}$ In this technique, an endoscope is introduced into the stomach and then a needle-knife or sphincterotome is used to puncture its anterior wall. The puncture can then be dilated with a pyloric dilating balloon. ${ }^{27,29}$ Once access to the peritoneal cavity has been accomplished, the procedure of choice is performed and the gastrotomy is closed. Several novel techniques are being developed to facilitate this, including the 
use of multiple conventional clips as well as experimental endoscopic suturing devices and closure over a percutaneous endoscopic gastrostomy (PEG) tube. ${ }^{29}$

Unfortunately, the majority of human studies to date have looked primarily at the technical feasibility of NOTES rather than its overall safety and efficacy. Animal models, especially porcine models, are promising but are limited by their physiologic differences to humans. ${ }^{29}$ Similarly, the human studies have largely involved very small numbers of patients, making them significantly underpowered to detect differences in viscerotomy and closure techniques. ${ }^{29}$

Overall, the complication rate from NOTES in human studies has varied considerably with reports ranging from $0 \%$ to $33 \%{ }^{30}$ These have ranged in severity from simple wound infections to more serious complications such as biliary leakage and ureteric injuries, and are likely related to both the procedure performed and the access site. How this compares to more conventional laparoscopic procedures remains an area of active study. In the recent RCT by Bulian et al, a complication rate of $10 \%$ was reported for both the control patients undergoing needlescopic cholecystectomy as well as the patients undergoing NOTES cholecystectomy. The two complications involving more conventional transabdominal laparoscopic techniques were both wound infections, whereas the complications from NOTES were both biliary pancreatitis. ${ }^{34}$

Despite significant early enthusiasm, NOTES cannot presently be recommended to routine clinical practice. While cosmetically appealing, NOTES should presently remain limited to well-designed experimental protocols or appropriately powered randomized clinical trials.

\section{Conclusion}

Since its birth in the 1980s, laparoscopic surgery has become a primary tool in the skill set of many abdominal surgeons. Despite its efficacy and widespread use, however, it is still associated with injuries to both visceral and vascular structures, many of which occur at entry into the abdominal cavity. Of the available techniques, the open Hasson approach appears to be the safest, though good results have also been seen using Veress needles and optical trocars. In addition, technological advances have led to the development of newer techniques, such as SILS and NOTES. Although technically feasible, both approaches remain largely experimental and have not yet been definitively shown to be superior to standard laparoscopy in regards to operative time and complication rates. Nevertheless, as these technologies are further refined, laparoscopic access techniques will continue to be modified in an attempt to increase efficacy, and most importantly, increase patient safety by minimizing complications.

\section{Disclosure}

The Division of General Surgery and Department of Surgery at the Ottawa Hospital receive unrestricted educational funding from Covidien Canada. G Martel has previously received a clinical and research bursary from Covidien Canada. The authors report no other conflicts of interest in this work.

\section{References}

1. Shabanzadeh DM, Sørensen LT. Laparoscopic surgery compared with open surgery decreases surgical site infection in obese patients: a systematic review and meta-analysis. Ann Surg. 2012;256(6): 934-945.

2. Ahmad G, O'Flynn H, Duffy JM, Phillips K, Watson A. Laparoscopic entry techniques. Cochrane Database Syst Rev. 2012;2:CD006583.

3. Nguyen NT, Scott-Conner CEH. The SAGES Manual. Berlin, Germany: Springer; 2012.

4. Vilos GA, Ternamian A, Dempster J. Laparoscopic entry: a review of techniques, technologies, and complications. J Obstet Gynaecol Can. 2007;29(5):433-465.

5. Hasson HM, Rotman C, Rana N, Kumari NA. Open laparoscopy: 29-year experience. Obstet Gynecol. 2000;96(5 Pt 1):763-766.

6. Bonjer HJ, Hazebroek EJ, Kazemier G. Open versus closed establishment of pneumoperitoneum in laparoscopic surgery. Br J Surg. 1997;84(5):599-602.

7. Larobina M, Nottle P. Complete evidence regarding major vascular injuries during laparoscopic access. Surg Laparosc Endosc Percutan Tech. 2005;15(3):119-123.

8. Chapron CM, Pierre F, Lacroix S, Querleu D. Major vascular injuries during gynecologic laparoscopy. J Am Coll Surg. 1997;185(5):461-465.

9. Schäfer M, Lauper M, Krähenbähl L. Trocar and Veress needle injuries during laparoscopy. Surg Endosc. 2001;15(3):275-280.

10. Berch BR, Torquati A, Lutfi RE, Richards WO. Experience with the optical access trocar for safe and rapid entry in the performance of laparoscopic gastric bypass. Surg Endosc. 2006;20(8):1238-1241.

11. String A, Berber E, Foroutani A, Macho JR, Pearl JM, Siperstein AE. Use of the optical access trocar for safe and rapid entry in various laparoscopic procedures. Surg Endosc. 2001;15(6):570-573.

12. Hallfeldt KK, Trupka A, Kalteis T, Stuetzle H. Safe creation of pneumoperitoneum using an optical trocar. Surg Endosc. 1999;13(3): 306-307.

13. Froghi F, Sodergren MH, Darzi A, Paraskeva P. Single-incision laparoscopic surgery (SILS) in general surgery: a review of current practice. Surg Laparosc Endosc Percutan Tech. 2010;20(4):191-204.

14. Gumbs AA, Milone L, Sinha P, Bessler M. Totally transumbilical laparoscopic cholecystectomy. J Gastrointest Surg. 2009;13(3):533-534.

15. Zhu JF, Hu H, Ma YZ, Xu MZ. Totally transumbilical endoscopic cholecystectomy without visible abdominal scar using improved instruments. Surg Endosc. 2009;23(8):1781-1784.

16. Hong TH, You YK, Lee KH. Transumbilical single-port laparoscopic cholecystectomy. Surg Endosc. 2009;23(6):1393-1397.

17. Mutter D, Leroy J, Cahill R, Marescaux J. A simple technical option for single-port cholecystectomy. Surg Innov. 2008;15(4):332-333.

18. Petrotos AC, Molinelli BM. Single-incision multiport laparoendoscopic (SIMPLE) surgery: early evaluation of SIMPLE cholecystectomy in a community setting. Surg Endosc. 2009;23(11):2631-2634.

19. Navarra G, Pozza E, Occhionorelli S, Carcoforo P, Donini I. One-wound laparoscopic cholecystectomy. Br J Surg. 1997;84(5):695.

20. Schlager A, Khalaileh A, Shussman N, et al. Providing more through less: current methods of retraction in SIMIS and NOTES cholecystectomy. Surg Endosc. 2010;24(7):1542-1546. 
21. Cuesta MA, Berends F, VeenhofAAFA. The "invisible cholecystectomy": a transumbilical laparoscopic operation without a scar. Surg Endosc. 2008;22(5):1211-1213.

22. Pelosi MA. Laparoscopic appendectomy using a single umbilical puncture (minilaparoscopy). J Reprod Med. 1992;37(7): 588-594.

23. Ateş O, Hakgüder G, Olguner M, Akgür FM. Single-port laparoscopic appendectomy conducted intracorporeally with the aid of a transabdominal sling suture. J Pediatr Surg. 2007;42(6): 1071-1074.

24. Carter JT, Kaplan JA, Nguyen JN, Lin MYC, Rogers SJ, Harris HW. A prospective, randomized controlled trial of single-incision laparoscopic vs conventional 3-port laparoscopic appendectomy for treatment of acute appendicitis. J Am Coll Surg. 2014;218(5):950-959.

25. Luna RA, Nogueira DB, Varela PS, et al. A prospective, randomized comparison of pain, inflammatory response, and short-term outcomes between single port and laparoscopic cholecystectomy. Surg Endosc. 2013;27(4):1254-1259.

26. Rabischong B, Compan C, Savary D, et al. La laparoscopie par incision unique en gynécologie: états des lieux en 2013 [Laparoendoscopic single-site surgery in gynecology: the situation in 2013]. J Gynecol Obstet Biol Reprod (Paris). 2013;42(5):445-457. French.

27. Flora ED, Wilson TG, Martin IJ, O’Rourke NA, Maddern GJ. A review of natural orifice translumenal endoscopic surgery (NOTES) for intraabdominal surgery. Ann Surg. 2008;247(4):583-602.
28. Giday SA, Kantsevoy SV, Kalloo AN. Principle and history of natural orifice translumenal endoscopic surgery (NOTES). Minim Invasive Ther Allied Technol. 2006;15(6):373-377.

29. McGee MF, Rosen MJ, Marks J, et al. A primer on natural orifice transluminal endoscopic surgery: building a new paradigm. Surg Innov. 2006;13(2):86-93.

30. Clark MP, Qayed ES, Kooby DA, Maithel SK, Willingham FF. Natural orifice translumenal endoscopic surgery in humans: a review. Minim Invasive Surg. 2012;2012:189296.

31. Hazey JW, Narula VK, Renton DB, et al. Natural-orifice transgastric endoscopic peritoneoscopy in humans: Initial clinical trial. Surg Endosc. 2008;22:16-20.

32. Tsin DA, Colombero LT, Lambeck J, Manolas P. Minilaparoscopyassisted natural orifice surgery. JSLS. 2007;11(1):24-29.

33. Gumbs AA, Fowler D, Milone L, et al. Transvaginal natural orifice translumenal endoscopic surgery cholecystectomy: early evolution of the technique. Ann Surg. 2009;249(6):908-912.

34. Bulian DR, Knuth J, Cerasani N, Sauerwald A, Lefering R, Heiss MM. Transvaginal/transumbilical hybrid-NOTES-versus 3-trocar needlescopic cholecystectomy: short-term results of a randomized clinical trial. Ann Surg. Epub October 28, 2014.
Open Access Surgery

\section{Publish your work in this journal}

Open Access Surgery is an international, peer-reviewed, open access journal that focuses on all aspects of surgical procedures and interventions. Patient care around the peri-operative period and patient outcomes post surgery are key topics. All grades of surgery from minor cosmetic interventions to major surgical procedures are covered. Novel techniques

Submit your manuscript here: http://www.dovepress.com/open-access-surgery-journal

\section{Dovepress}

and the utilization of new instruments and materials, including implants and prostheses that optimize outcomes constitute major areas of interest. The manuscript management system is completely online and includes a very quick and fair peer-review system. Visit http://www.dovepress.com/ testimonials.php to read real quotes from published authors. 